\title{
A Multiuser Multiperspective Stereographic QTVR Browser Complemented by Java3D Visualizer and Emulator
}

Michael Cohen, Noor Alamshah Bolhassan, and Owen Noel Newton Fernando 
Spatial Media Group, Computer Arts Lab.

University of Aizu

Aizu-Wakamatsu, Fukushima-ken 965-8580

Japan

mcohen@u-aizu.ac.jp

voice: [+81](242)37-2537

fax: [+81](242)37-2772

Second author's current address:

Faculty of Computer Science and Information Technology

Universiti Malaysia Sarawak

94300 Kota Samarahan

Sarawak, Malaysia

bnalamshah@fit.unimas.my

Third author's current address:

Mixed Reality Lab., Dept. of Electrical and Computer Engineering

National University of Singapore

7 Engineering Drive 1, Blk E3A, \#02-04/05

Singapore 117574

newtonfernando@mixedrealitylab.org

Note the use of emboldening and small caps, as in 2D, 2.5D, 3D, 3D-CGI, Java3D) to denote acronyms: (CAD, CAT [computer axial tomography], CMOS, GIF, MIDlets) and initials AR [augmented reality], (CDR [computed dental radiography], CG, CGI, CPU, collaborative virtual environments (CVES), CW and CCW, DV [digital video], "first-person shooter" (FPS), HMDs, 
IBR [image-based rendering], J3D, JME, JMF, JSE, liquid-crystal display (LCD), MR [mixed reality], QTVR, SQTVR. Also note the exotic orthography of $\mathrm{VR}_{4} \mathrm{U}_{2} \mathrm{C}$, the designation of keys as in Control, Shift, \& Alt $+, \leftarrow, \rightarrow, \uparrow, \&, \downarrow$; the use of monospacing to indicate computer modes and keywords, anaglyphic, parallel; CVEClientIF, get and set; parallel, cross-eyed, over/under; point 1 etc.; "View 0" etc. Also note copious use of footnotes of URLs for commercial web pages, for which including the access date (as suggested by the Presence style guide) would be inappropriate. 


\begin{abstract}
To support multiperspective and stereographic image display systems intended for multiuser applications, we have developed two integrated multiuser multiperspective stereographic “browsers," respectively featuring IBR-generated egocentric and CG exocentric perspectives. The first one described, “ $V R_{4} U_{2} C$ ” ( 'virtual reality for you to see'), uses Apple's QuickTime VR technology and the Java programming language together with the support of the 'QuickTime for Java' library. This unique QTVR browser allows coordinated display of multiple views of a scene or object, limited only by the size and number of monitors or projectors assembled around or among users (for panoramas or turnoramas) in various viewing locations. The browser also provides a novel solution to limitations associated with display of QTVR imagery: its multinode feature provides interactive stereographic QTVR (dubbed 'SQTVR') to display dynamically selected pairs of images exhibiting binocular parallax, the stereoscopic depth percept enhanced by motion parallax from displacement of the viewpoint through space coupled with rotation of the view through a $360^{\circ}$ horizontal panorama. This navigable approach to SQTVR allows proper occlusion/disocclusion as the virtual standpoint shifts, as well as natural looming of closer objects compared to more distant ones. We have integrated this stereographic panoramic browsing application in a client/server architecture with a sibling client, named “Just Look at Yourself!," which is built with Java3D and allows realtime visualization of the dollying and viewpoint adjustment as well as juxtaposition and combination of stereographic CG and IBR displays. Just Look at Yourself! visualizes and emulates $V R_{4} U_{2} C$, embedding avatars associated with cylinder pairs wrapped around the stereo standpoints texture-mapped with a set of panoramic scenes into a 3D CG model of the same space as that captured by the set of panoramas. The transparency of the 3D CG polygon space and the photorealistic stereographic $360^{\circ}$ scenes, as well as the size of the
\end{abstract}

